\title{
Maßnahmen zur Qualitätsförderung in der Arztpraxis
}

\author{
Jeder Arzt ist entsprechend seiner Berufsordnung verpflichtet, Maßnahmen zur Sicherung der \\ Qualität medizinischer Versorgung durchzuführen. Diese sollen messbar sein und der konkreten Er- \\ reichung eines festgelegten Ziels dienen. Im allgemeinen Sprachgebrauch wird die Verwendung des \\ Begriffs "Qualität" meist mit "guter" oder "bester" Leistung assoziiert. Ärztlichem Handeln wird also \\ implizit eine höchste Qualität unterstellt.
}

Die Bundesregierung hat mit der Gesundheitsreform 2004 alle niedergelassenen Ärzte und Psychotherapeuten verpflichtet, ab dem 01.01.2006 ein praxisinternes Qualitätsmanagement (QM) einzuführen. Auch Ärzte, die in einem Krankenhaus in Bereichen arbeiteten, in denen noch kein QM-System eingebettet ist, müssen sich daran beteiligen.

Durch QM kann der Arzt den Wert seiner Praxis steigern und für eventuelle Nachfolger attraktiver gestalten. Zudem erhält der Arzt Rückmeldungen von seinen Patienten. Es unterstützt ihn in der Überprüfung und Sicherung der Zukunftsfähigkeit seines Behandlungskonzepts und somit seiner Arztpraxis. Ein funktionierendes QM vermeidet zudem Doppelarbeit.

QM muss von der Praxisleitung initiiert werden. Die Umsetzung bezieht jedoch alle Mitarbeiter der Praxis mit ein. Da es zwischen Kosten und Qualität einen Zusammenhang gibt, findet QM immer auch unter wirtschaftlichen Gesichtspunkten statt. Dient QM lediglich zur Erfüllung der gesetzlichen Vorgaben, kann es als zeitintensiv und überflüssig empfunden werden. Aktiv betrieben, stellt es jedoch ein hervorragendes "Controlling"- und Steuerungsinstrument dar. Es ist daher sinnvoll, die Mitarbeiter vom QM zu überzeugen und es als Vorgesetzter selbst anzuwenden. Wann eine abschließende Zertifizierung vorgenommen wird, muss letztendlich jede Praxis selbst entscheiden.

\section{Müssen Ärzte bestimmte Fristen beachten?}

Die Einführung des QM gliedert sich in drei Phasen: In der Planungsphase haben Ärzte zunächst zwei Jahre lang Zeit, das QM-System für ihre Praxis vorzubereiten und einzuführen. Während der anschließenden Umsetzungsphase in den folgenden zwei Jahren wenden sie das QM-System an. Nach einem weiteren Jahr, der Überprüfungsphase, müssen Sie den Stand der Umsetzung danach bewerten, inwiefern Sie ihre anvisierten Ziele erreicht haben.

\section{Müssen Ärzte von sich aus die Einführung eines QM-Systems nachweisen?}

Nein. Die QM-Kommission fordert in einer jährlichen Stichprobe Arztpraxen auf, den Einführungs- und Entwicklungsstand ihres QMSystems schriftlich anzugeben. Falls daraus hervorgeht, dass, verglichen mit der Richtlinie, der Einführungsstand noch nicht erfolgt ist, berät die QM-Kommission in einem kollegialen Gespräch mit dem Arzt Schritte, wie er in einem gemeinsam festgelegten Zeitraum den erforderlichen Stand erreichen kann. Außerdem erstellt die Krankenversicherung jährlich einen Bericht über den Einführungsstand im Bezirk.

\section{Wie findet man das richtige QM-System?}

Ein bestimmtes QM-System ist für Praxen gesetzlich nicht vorgeschrieben. Es muss lediglich die Bereiche „Patientenversorgung" und „Praxisführung" abdecken.

Bei der Patientenversorgung soll die medizinische Versorgung u.a. den fachlichen Standards und Leitlinien auf aktuellem Forschungsstand entsprechen. Bei der Praxisführung geht es darum, neben regelmäßiger Mitarbeiterfortbildung auch Terminplanung, Datenschutz, Hygiene sowie die Kooperation mit Nahtstellen der medizinischen Versorgung - etwa Apotheken oder Physiotherapeuten - zu fördern.

Nach erfolgreicher externer Überprüfung der Qualitätsmaßnahmen anhand eines vorher gewählten QM-Systems durch einen Auditor wird ein Zertifikat vergeben, das die Einhaltung von Standards dokumentiert. So wird zum Beispiel durch eine erfolgreiche Zertifizierung nach dem System „Qualität und Entwicklung in Praxen ${ }^{\circledR}$ (QEP) das „Deutsche Gesundheitssiegel" vergeben.

Die Ärztliche Zentralstelle Qualitätssicherung (ÄZQ) stellt im „Kompendium Q-M-A“ umfangreiche Informationen zu verschiedenen QM-Systemen zur Verfügung. Es kann auf www.aezq.de heruntergeladen werden.

\section{Korrespondenzadresse \\ Monika Dumont \\ Leitung Kommunikation und Marketing \\ Duxxess - Gesellschaft für medizinisches Erfolgsmanagement $\mathrm{mbH}$ monika.dumont@duxxess.com}

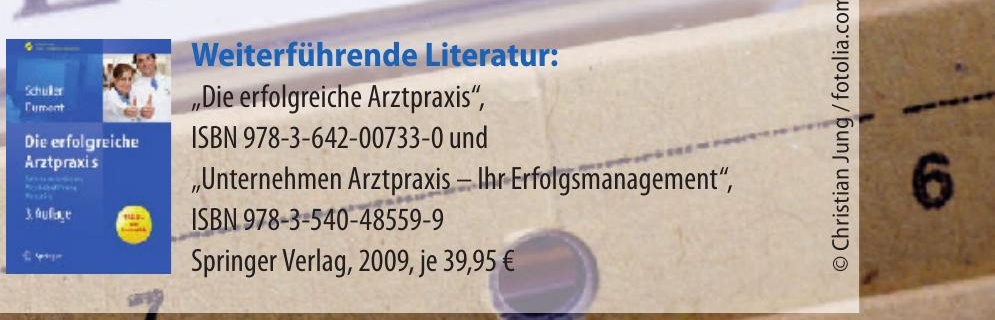

